\title{
ANALYSING THE EFFECTIVENESS OF ONLINE GAMIFIED LEARNING METHODS AS OPPOSED TO TRADITIONAL LEARNING METHODS FOR TEACHING COMPUTER PROGRAMMING
}

\author{
Owen Sacco \\ Malta College of Arts, Science and Technology, Malta
}

\begin{abstract}
Traditional teaching methods for teaching computer programming suffer from keeping students engaged and motivated to learn how to code. On the other hand, video games have a very high successful rate of keeping players motivated and engaged for hours without loss of focus to solve a set of tasks without feeling fatigued or frustrated. In this regard, in this research we analyse the effectiveness of gamified learning methods compared to traditional learning methods for teaching computer programming by conducting a study where students are taught computer programming using both teaching methods. The results from our user study are presented in this paper.
\end{abstract}

\section{KEYWORDS}

Traditional-Based Learning, Gamified-Based Learning, Computer Programming Education

\section{INTRODUCTION}

The main research study of this work is to examine the effectiveness of online gamified learning as opposed to traditional taught methods when teaching computer programming. Currently, students struggle to learn computer programming and find the topic hard to grasp (Olsson, et al., 2015; Lahtinen, et al., 2005). The main problem consists of students are not motivated and engaged in the subject, which results in students not practicing computer coding enough in order for them to learn the subject. Moreover, traditional taught methods are not feasible at keeping students motivated and engaged for learning computer programming. In this regard, other methods such as online gamified learning methods need to be employed to motivate students to code more and be more immersed in the subject. Video games are very successful in keeping players immersed, motivated and engaged for hours without loss of focus to accomplish tasks set out in the game (Green \& Bavelier, 2006). Moreover, in games, players solve complex tasks within virtual environments without feeling fatigued or frustrated, compared to traditional taught learning methods where students struggle to solve computer programming tasks. Gamified learning methods therefore consist of integrating game elements in teaching methods in order to increase students' motivation and engagement whilst learning computer programming. Through this research, an analysis will be conducted to study the effectiveness of employing online gamified methods to examine whether students' motivation, engagement, attention and retention are improved through play.

Several online gamified platforms, such as CodinGame ${ }^{1}$, provide users to learn coding through fun by completing tasks similar to tasks in games. Mainly, users are requested to solve specific game-like problems through code and are rewarded accordingly after completing successfully the task.

This research consists of first analysing students' behaviour through traditional methods - the students were taught specific coding concepts using traditional methods and afterwards were given coding tasks to complete. Once the coding tasks were complete, the students were asked to fill-in a survey about their learning experience using traditional methods. The research then analysed the students' behaviour through online gamified methods

${ }^{1}$ CodinGame - https://www.codingame.com/ 
- the students were then introduced to online gamified platforms and were asked to complete tasks on this platform. Once the students completed the gamified tasks, the students were asked to fill-in a survey about their learning experience using the online gamified platform. Ultimately, the survey results from both methods were compared to analyse which teaching methods students preferred and also to analyse whether students' motivation, engagement, attention and retention improved using online gamified methods. The results of such study are provided in this work. The remainder of this paper is as follows: Section 2 offers core information about gamification and reviews current work in this area. Section 3 provides an explanation of the different teaching methods used for our analysis in this work and Section 4 presents in detail our results from our user study. Section 5 concludes the paper by providing an overall discussion and the future steps of this work.

\section{RELATED WORK}

Teaching computer programming requires abstract thinking and problem-solving skills apart from learning the computer programming lanague, which constantly poses a challenge for educators to teach such a subject (Piteira, et al., 2018). Normally, when students are presented with complex tasks to solve using computer programming, studennts are overwhelmed and since they do not receive any immediate saisfaction or short term wins, they end up feeling unengaged, unmotivated and thus, loose attention in class (Fotaris, et al., 2016). However, when players are presented with complex tasks to solve in video games, players are kept at a high level of attention, last for many hours and strive until they solve the tasks and win (Green \& Bavelier, 2006). In this regard, several studies have been proposed to incorporate game-elements in educational context, in particular in teaching computer programming (Costa, et al., 2017; Ibanez, et al., 2014), in order to increase students' attention, engagement, motivation and retention.

Gamification involves adding game elements to non-game context (Deterding, et al., 2011). Game-elements in a non-game setting consist of the use of points, medals, levels, progress bars, leader-boards, amongst others (Deterding, et al., 2011). Gamification in educational environments can provide an increase to students' engagement in learning whilst providing instant feedback by indicating the player whether s/he won or not (Seaborn \& Fels, 2015). In a gamified environment, students can learn by completing activities and also learn through error which are represented as achievements, medals, honour board, etc. (Richards, et al., 2014). Gamification provides an environment where learning is rewarded and allows students to assess their skills and abilities. Moreover, through gamification, students may see failure as a learning opportunity (Piteira, et al., 2018) and it encourages them to redo the task until the students grasps the concepts and ultimately manage to solve the task. In (Borys \& Laskowski, 2013) the authors identified 36 learning principles that video games could benefit as a learning tool in education, which included immediate feedback, self-learning, information on demand, collaborative learning amongst others. Thus, current literature illustrates the imortance of applying gamification to teaching methods since it could motivate and engage students to learn more. Although several research have studied the use of gamification in education, a more in-depth research is required to study the effectiveness of incorporating gamification in teaching methods (Dicheva, et al., 2015). In this regard, this motivated the research study area of this paper.

The most closest study to the research presented in this paper is the work in (Fotaris, et al., 2016). In this work, the authors study the effects of gamification using the following applications: Kahoot! ${ }^{2}$, an open-source adaptation of Who Wants to be a Millionaire, and Codecademy. Although these methods provide gamified environments, students are not provided with game-like tasks when writing code. Therefore, in our research study, we opted to use a platform that provides game-like tasks to analyse the engagement and motivation of students whilst solving tasks through writing code since as mentioned above, video games should incrase students' attention, motivation, and should encourage them to strive until they solve the tasks and win (Green \& Bavelier, 2006). In this regard, CodinGame is chosen as the platform to conduct the study in this paper to assess students' engagement, motivation, attention and retention in learning computer programming through solving game-like tasks.

\footnotetext{
${ }^{2}$ Kahoot! - https://kahoot.com/
} 


\section{METHODOLOGY}

The user study was conducted on a 16 -week $2^{\text {nd }}$ year undergraduate study unit in which students learn how to program using the $C \#$ programming language. The total amount of students following this unit is around 70 students. In this unit, lectures cover fundamental programming concepts ranging from data types and variables, selection, arrays and loops, methods, classes and objects (object-oriented paradigm), encapsulation and abstraction, polymorphism, inheritance, interfaces, exception handling, graphical user interfaces and databases. As part of the user study, two main teaching methods were used to introduce different programming concepts in order to analyse which method was most effective and which method students preferred. The two main teaching methods are the traditional teaching methods and the gamified teaching methods. First, the traditional teaching methods was used and afterwards the students were asked to fill-in a survey about their learning experience using traditional methods. Subsequently, gamified teaching methods were used and students were asked to fill in a survey to gain insights about their learning experience using gamified methods.

This section provides an explanation of the different teaching methods used in our user study for analysing the effectiveness of online gamified teaching methods for teaching computer programming in class.

\subsection{Traditional Teaching Methods}

Traditional teaching methods are the most common type of teaching methods used in class for teaching computer programming courses. Traditional teaching methods for teaching computer programming consist of lecture-type methods, live-coding type methods and task-based type methods.

Lecture-type teaching methods consist of the lecturer presenting computer programming concepts using slides with minimum amount of programming in class. Students must attentively listen to what the lecturer is explaining, and try to formulate an understanding of how such concepts are applied in computer programs. The students ask questions based on the concepts presented in class rather than on any queries which they might encounter whilst applying these concepts when coding. At the end of the lecture, students are given review tasks based on the concepts presented in class to work out of class. Students work individually on these tasks out of class and have to solve the tasks based on what they have learnt during the lecture without having the lecturer present to answer any of their queries which they might encounter whilst coding.

Live-coding type methods consist of the lecturer presents computer programming concepts by live-coding examples in class and the students follow the lecturer by coding (i.e. copying) what the lecturer has just coded. With this method, the students are not only learning the concepts but can visualize better how these concepts can be applied in computer programs. With this method the students have the opportunity to ask questions not only about the concepts presented but also any other queries which the students encounter whilst programming. At the end of the lecture, the students are given review tasks to work out outside of class. Yet again, students must work these tasks individually out of class and have to solve the tasks without having the lecturer present to answer any of their queries which they might encounter whilst working out these tasks

Task-based type methods consist of hands-on practical sessions whereby most of the lectures consist of the students being given practical tasks to work out in class and they learn programming concepts as they solve the tasks and progress from one task to another. The lecturer in this type of teaching method is more of a facilitator whereby the lecturer is only present to answer any queries and ensures that the students are progressing from one task to another. Thus, in this type of teaching method, the students learn by coding themselves in class.

\subsection{Gamified Teaching Methods}

Gamified teaching methods consist of teaching computer programming concepts with game-elements in order to engage and motivate the students to learn programming concepts through fun and play. An online gamified platform called CodinGame ${ }^{3}$ is used during these sessions whereby students are given practical tasks to solve which are related to the programming concept being taught. These are presented in the form of a quest or task in a game. The students are provided with the goal which they have to reach and are also given rules about the

${ }^{3}$ CodinGame - https://www.codingame.com/ 
game. The students must then program their task based on the rules provided in order to reach the goal. In the end, they could test their code and see their solution being animated. The platform indicates whether they have managed to solve the task by reaching the goal and it also indicates in which part the students failed. When students complete the task successfully, they unlock achievements, gain experience points and are provided with their score. Moreover, students could compete against each other in a leader board which shows the scores of each player sorted from highest to lowest. In this way, students are incentivised similar in games to be better and beat their "opponents" (opponents being their classmates) in the leader board.

\section{EVALUATION - USER STUDY}

This section presents the results of our user study. The responses follow a five point Likert scale: 1 - Strongly Disagree, 2 - Disagree, 3 - Undecided, 4 - Agree, and 5 - Strongly Agree.

The survey first asked the participant's gender and we observe that $80 \%$ are male, $15 \%$ are female and $5 \%$ prefer not to say.

The survey then asked the participants what is their frequency at playing video games. This is to determine their level at playing digital games. We observe that $42 \%$ play video games frequently, $16 \%$ play video games often, $16 \%$ sometimes play video games, $21 \%$ play video games occasionally and only $5 \%$ never play video games. These results depict that most of the participants know how to play video games.

\subsection{Traditional Teaching Methods}

This section presents the results from the participants' experience when learning computer programming through traditional teaching methods. The traditional teaching methods include lecture-type teaching methods, live-coding type teaching methods and task-based type teaching methods as explained in section 3.

Q1 - Motivates Learning: participants were asked whether traditional teaching methods motivate them to learn computer programming. Figure 1 depicts that most of the participants are unsure whether traditional

teaching methods motivate them in learning computer programming whereas only a few strongly agree that these methods motivate them.

Q2 - Fun and Engaging: participants were asked whether traditional teaching methods are fun and engaging. Figure 2 depicts that most participants disagree that traditional teaching methods are fun and engaging and only a few strongly agree that these methods are in fact fun and engaging.

Q3 - Sufficient Material: participants were asked whether traditional teaching methods provide enough material for the students to learn computer programming. Figure 3 illustrates that most participants agree that these methods indeed provide sufficient material for the students to learn computer programming.

Q4 - Assignment / Exam Preparation: participants were asked whether traditional teaching methods prepares them well enough for an assignment and/or exam. Figure 4 depicts that most participants agree that through these methods they feel that they are prepared enough for an assignment and/or exam, and only a few do not feel prepared well enough for an assignment and/or exam.

Q5 - Motivates Research: participants were asked whether traditional teaching methods encourages them to research more about the computer concepts explained in class. Figure 5 illustrates that most participants do not feel encouraged to research more about programing concepts whereas $35 \%$ feel motivated to research more.

Q6 - Motivate Attention in Class: participants were asked whether traditional teaching methods motivates them to be more attentive during class. Figure 6 illustrates that the traditional teaching methods do not motivate students to be attentive in class. This could be linked with $Q 2$ above since they do not find traditional teaching methods as fun and engaging.

Q7 - Motivates Class Attendance: participants were asked whether traditional teaching methods motivates them to attend class. Figure 7 illustrates that students are not motivated to attend class when computer programming is taught through traditional teaching methods. Similar to $Q 6$, this could be correlated with the students not finding traditional teaching method as fun and engaging as noted in $Q 2$.

Q8 - Motivates Computer Programming Career: participants were asked whether traditional teaching methods motivates them to choose a computer programming career. Figure $\mathbf{8}$ illustrates that the majority of students are undecided, whilst $20 \%$ of students agree and another $20 \%$ of students strongly agree that traditional teaching methods motivates them to choose a computer programming career in the future. 


\subsection{Gamified Teaching Methods}

This section presents the results from the participants' experience when learning computer programming through gamified teaching methods. The gamified teaching methods consist of teaching computer programming concepts with game-like tasks using the online gamified platform called CodinGame ${ }^{4}$ as explained in section 3 .

Q1 - Motivates Learning: participants were asked whether gamified teaching methods motivate them to learn computer programming. Figure 1 depicts that most of the students agree and strongly agree that they are motivated in learning computer programming through gamified teaching methods whereas only a few disagreed.

Q2 - Fun and Engaging: participants were asked whether gamified teaching methods are fun and engaging. Figure $\mathbf{2}$ depicts that the majority of the participants find gamified teaching methods as fun and engaging and only a few do not find gamified teaching methods as fun and engaging.

Q3 - Sufficient Material: participants were asked whether gamified teaching methods provide enough material for the students to learn computer programming. Figure 3 illustrates that the majority of students agree and strongly agree that these methods indeed provide sufficient material for the students to learn computer programming. The online gamified platform provides a learn section which contains many pages with learning content that explains most of the computer programming concepts. The platform provides learning content for a substantial amount of different programming languages. This means that students may not only learn the C\# programming language, but may also learn other programming languages through this platform.

Q4-Assignment / Exam Preparation: participants were asked whether gamified teaching methods prepares them well enough for an assignment and/or exam. Figure $\mathbf{4}$ depicts that most participants are undecided whether gamified teaching methods prepares them well for assessments. The reason for this could be since the gamified teaching methods is still new for the students and this user study was conducted prior to students attempting any assessment. Therefore, the students could not judge whether through gamified learning methods they are well prepared or not for assessments.

Q5 - Motivates Research: participants were asked whether gamified teaching methods encourages them to research more about the computer concepts explained in class. Figure 5 illustrates that $30 \%$ of the students strongly agree and $20 \%$ of the students agree that they feel encouraged to research more about programing concepts whereas $35 \%$ are undecided whether this method encourages them to research more about the computer programming concepts covered in class.

Q6 - Motivate Attention in Class: participants were asked whether gamified teaching methods motivates them to be more attentive during class. Figure $\mathbf{6}$ illustrates that the majority of the students feel motivated to remain attentive during class. This could be correlated with $Q 2$ above since they find gamified teaching methods as fun and engaging.

Q7 - Motivates Class Attendance: participants were asked whether gamified teaching methods motivates them to attend class. Figure 7 illustrates that gamified teaching methods motivates the majority of students to attend. Similar to $Q 6$, this could be correlated with $Q 2$ above whereby students find gamified teaching methods as fun and engaging.

Q8 - Motivates Computer Programming Career: participants were asked whether gamified teaching methods motivates them to choose a computer programming career. Figure $\mathbf{8}$ illustrates that most of the students agree and strongly agree that gamified teaching methods motivates them to choose a computer programming career in the future.

\subsection{Comparison of Results}

This section presents the comparison of results by comparing the results from the traditional teaching methods and the results from the gamified teaching methods, as illustrated in Table 1. In Table 1, for interpretation purposes, the strongly agree and the agree percentages have been added together to form a positive result, the undecided percentages remain unchanged and are represented by a neutral result, and the disagree and strongly disagree percentages have been added together to form a negative result.

${ }^{4}$ Codin Game - https://www.codingame.com/ 
Table 1. Comparison of Results

\begin{tabular}{lcccccc}
\hline \multirow{2}{*}{ Question } & \multicolumn{3}{c}{$\begin{array}{c}\text { Traditional Teaching } \\
\text { Methods }\end{array}$} & \multicolumn{3}{c}{ Gamified Teaching Methods } \\
& Positive & Neutral & Negative & Positive & Neutral & Negative \\
\hline Q1 - Motivates Learning & $35 \%$ & $45 \%$ & $20 \%$ & $\mathbf{6 5 \%}$ & $15 \%$ & $20 \%$ \\
Q2 - Fun and Engaging & $20 \%$ & $35 \%$ & $45 \%$ & $\mathbf{7 0 \%}$ & $15 \%$ & $15 \%$ \\
Q3 - Sufficient Material & $\mathbf{5 5 \%}$ & $25 \%$ & $20 \%$ & $\mathbf{5 5 \%}$ & $30 \%$ & $15 \%$ \\
Q4 - Assignment / Exam Preparation & $\mathbf{5 0 \%}$ & $30 \%$ & $20 \%$ & $30 \%$ & $\mathbf{5 0 \%}$ & $20 \%$ \\
Q5 - Motivates Research & $35 \%$ & $5 \%$ & $60 \%$ & $\mathbf{5 0 \%}$ & $35 \%$ & $15 \%$ \\
Q6 - Motivate Attention in Class & $30 \%$ & $25 \%$ & $45 \%$ & $\mathbf{6 0 \%}$ & $25 \%$ & $15 \%$ \\
Q7 - Motivates Class Attendance & $30 \%$ & $15 \%$ & $55 \%$ & $\mathbf{6 0 \%}$ & $30 \%$ & $10 \%$ \\
Q8 - Motivates Computer Programming Career & $40 \%$ & $40 \%$ & $20 \%$ & $\mathbf{6 0 \%}$ & $30 \%$ & $10 \%$ \\
\hline Mean & $37 \%$ & $28 \%$ & $36 \%$ & $\mathbf{5 6 \%}$ & $29 \%$ & $15 \%$ \\
\hline
\end{tabular}

Overall, it can be noted that students prefer more gamified teaching methods over traditional teaching methods with a mean positive feeling of $56 \%$ for gamified teaching methods as opposed to $37 \%$ positive feeling for traditional teaching methods. Moreover, gamified teaching methods gained a higher positive feeling in most of the results, except for the assignment and exam preparation. The students preferred more traditional teaching methods when it came to assignment and exam preparation but were neutral for gamified teaching methods. As explained in the above sections, this could be due to the fact that the survey was conducted before any assignment and/or exam was given to the students and therefore they could not judge whether gamified teaching methods prepare them well enough for any type of assessment. With regards to which teaching methods provide sufficient material, it can be noted that both the traditional teaching methods and the gamified methods provide sufficient material for the students to learn computer programming.

In the end, the survey concluded by asking the participants to select which teaching methods they preferred in certain scenarios. This occurred to reinforce the results which the students have selected for each teaching methods above. The following are the results of the students' preferences.

C1 - Preferred Teaching Methods in General: participants were asked which teaching methods they preferred in general in class and $60 \%$ preferred gamified teaching methods as opposed to $40 \%$ who preferred traditional teaching methods instead. These results are close to the mean positive results compared above $-56 \%$ for gamified teaching methods and $37 \%$ for traditional teaching methods.

C2 - Preferred Teaching Methods for Learning Computer Programming: participants were asked which teaching methods they preferred the most to learn computer programming and $65 \%$ preferred gamified teaching methods and $35 \%$ preferred traditional teaching methods. These results are also close to the mean positive results compared above.

C3 - Preferred Teaching Methods that makes Learning Computer Programming Easier: participants were asked which teaching methods makes learning computer programming easier and $70 \%$ have selected gamified teaching methods whereas $30 \%$ have selected traditional teaching methods.

C4 - Teaching Methods Most Fun and Engaging: participants were asked which teaching methods made learning computer programming fun and engaging, and $90 \%$ have selected gamified teaching methods as the most fun and engaging whereas only $10 \%$ have selected traditional teaching methods as fun and engaging. These results are in fact more than the overall positive results compared above where it was recorded that $70 \%$ are positive about gamified teaching methods being more fun and engaging as opposed to $20 \%$ being positive about traditional teaching methods being more fun and engaging.

C5 - Preferred Teaching Methods to Achieve Better Grades: participants were asked which teaching methods help them to achieve better grades - $60 \%$ have selected traditional training methods as the preferred teaching method that helps them to achieve better grades whereas $40 \%$ selected gamified teaching methods.

In conclusion, from this study, gamified teaching methods have obtained overall the most positive responses, which are also in-line with the results obtained in (Fotaris, et al., 2016) that use different gamification elements. However, from our results, $60 \%$ still feel that with traditional teaching methods they could achieve better grades and thus, one cannot adopt gamified only teaching methods in class but a blended approach should be adopted. In this regard, more research should be studied to explore new methods where gamified teaching methods would be the basis of course delivery for teaching computer programming but it should also incorporate traditional teaching methods in order to prepare students for their assessments. 
Therefore, a gamified traditional teaching method is required to improve student motivation, attention, information retention and learning development for learning computer programming.

\section{CONCLUSION}

In this work we have conducted a user study to analyse the effectiveness of gamified teaching methods through game-like coding tasks compared to traditional teaching methods, and from our findings we have concluded that overall, students prefer having gamified teaching methods. However, the results show that having a gamified only teaching method is not enough but a mixed teaching approach of traditional teaching methods combined with gamified teaching methods is required in order to prepare students in their assessments. In this regard, as future work, we will first analyse the impact gamified teaching methods using game-like problem solving coding tasks have to prepare students in their assessments, compared to when traditional teaching methods are adopted to prepare students in their assessments. Secondly, we will develop and incorporate a gamified teaching methods platform plugin, consisting of game-like problem solving coding tasks (similar to the CodinGame platform), within the Moodle virtual lecturing environment (VLE) as to our knowledge no such plugin containing gamified game-like coding tasks exist for Moodle. Ultimately, we will assess the effectiveness of incorporating such features into the VLE coupled with the course material that is shared with the students within this system by analysing whether students' motivation, engagement, learning development and retention increases.

\section{REFERENCES}

Borys, M. \& Laskowski, M., 2013. Implementing game elements into didactic process: A case study. s.l., Management, knowledge and learning international conference.

Costa, C. J., Aparicio, M., Aparicio, S. \& Aparicio, J. T., 2017. Gamification usage ecology. s.1., ACM International Conference on the Design of Communication.

Deterding, S., Dixon, D., Khaled, R. \& Nacke, L., 2011. From game design elements to gamefulness: defining" gamification. Proceedings of the 15th international academic MindTrek conference: Envisioning future media environments, pp. 9-15.

Dicheva, D., Dichev, C., Agre, G. \& Angelova, G., 2015. Gamification in education: A systematic mapping study. Journal of Educational Technology \& Society, 18(3).

Fotaris, P., Mastoras, T., Leinfellner, R. \& Rosunally, Y., 2016. Climbing up the Leaderboard: An Empirical Study of Applying Gamification Techniques to a Computer Programming Class. Electronic Journal of e-learning, 14(2), pp. 94-110.

Green, C. S. \& Bavelier, D., 2006. Effect of action video games on the spatial distribution of visuospatial attention.. Journal of experimental psychology: Human perception and performance, 32(6), pp. 1465-1478.

Ibanez, M.-B., Di-Serio, A. \& Delgado-Kloos, C., 2014. Gamification for engaging computer science students in learning activities: A case study. IEEE Transactions on learning technologies, 7(3), pp. 291-301.

Lahtinen, E., Ala-Mutka, K. \& Järvinen, H.-M., 2005. A study of the difficulties of novice programmers. Proceedings of the 10th Annual SIGCSE Conference on Innovation and Technology in Computer Science Education, 37(3), p. 14-18.

Olsson, M., Mozelius, P. \& Collin, J., 2015. Visualisation and Gamification of e-Learning and Programming Education.. Electronic journal of e-learning, 13(6), pp. 441-454.

Piteira, M., Costa, C. J. \& Aparicio, M., 2018. Computer programming learning: how to apply gamification on online courses?. Journal of Information Systems Engineering \& Management, 3(2).

Richards, C., Thompson, C. W. \& Graham, N., 2014. Beyond designing for motivation: the importance of context in gamification. s.l., Proceedings of the first ACM SIGCHI annual symposium on Computer-human interaction in play.

Ryan, R. M. \& Deci, E. L., 200. Intrinsic and extrinsic motivations: Classic definitions and new directions. Contemporary educational psychology, 25(1), pp. 54-67.

Seaborn, K. \& Fels, D. I., 2015. Gamification in theory and action: A survey. International Journal of human-computer studies, Volume 74, pp. 14-31. 


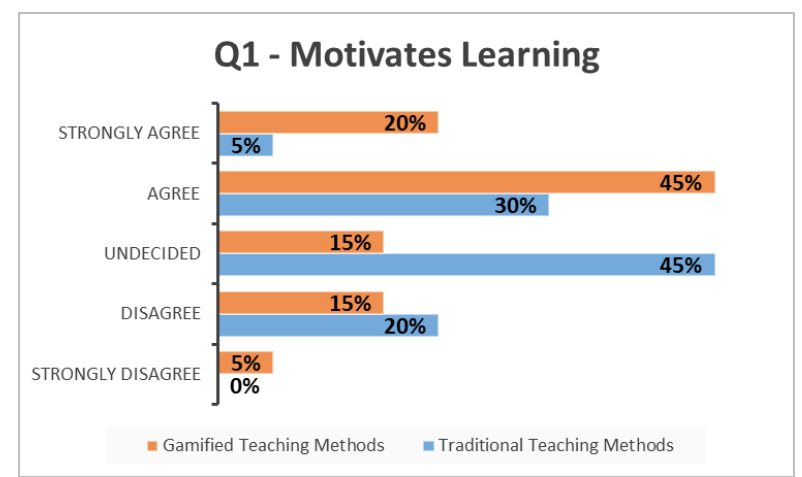

Figure 1. Q1 - Learning

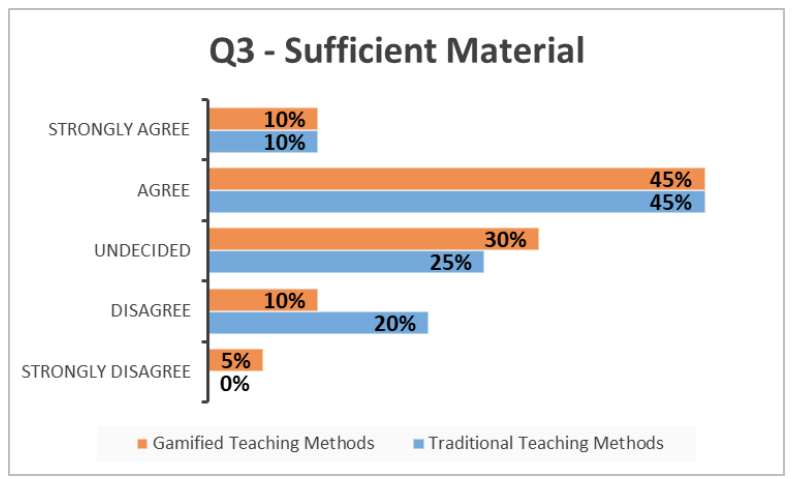

Figure 3. Q3 - Sufficient Material

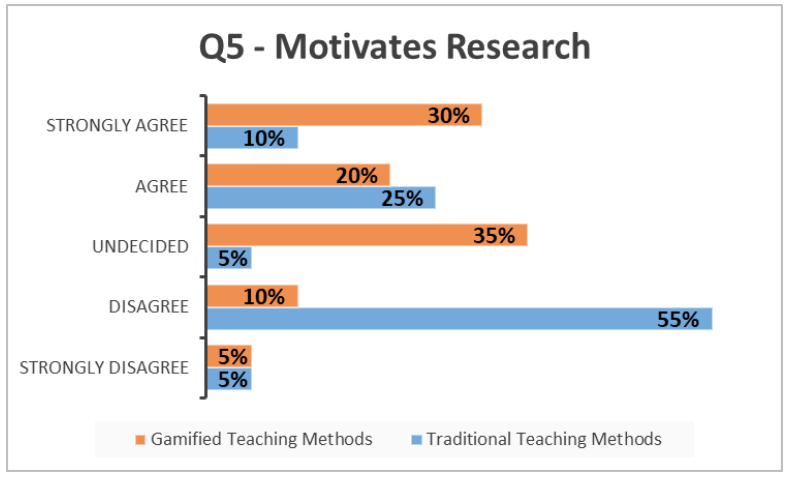

Figure 5. Q5 - Research

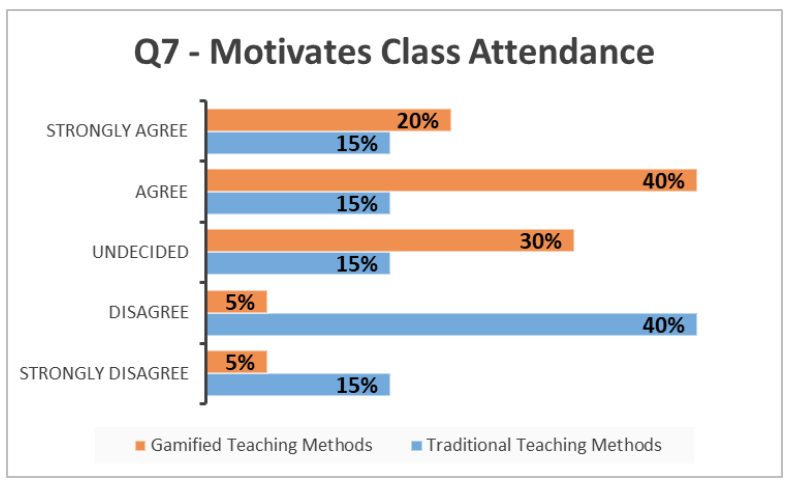

Figure 7. Q7 - Class Attendance

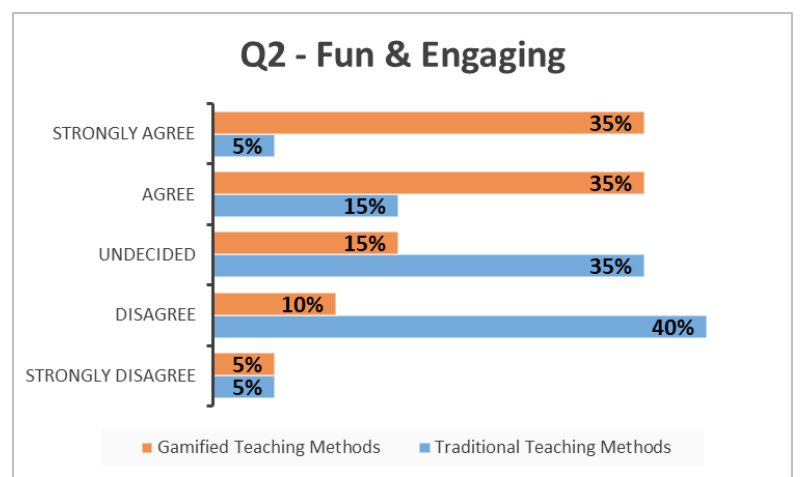

Figure 2. Q2 - Fun \& Engaging

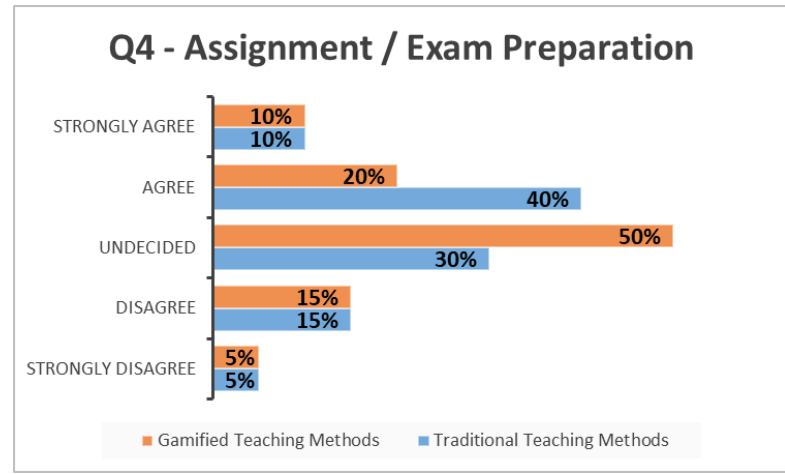

Figure 4. Q4 - Assignment / Exam Preparation

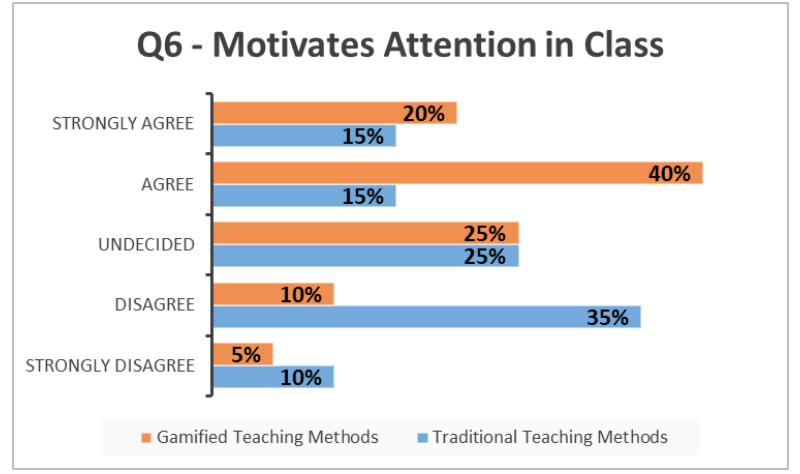

Figure 6. Q6 - Attention in Class

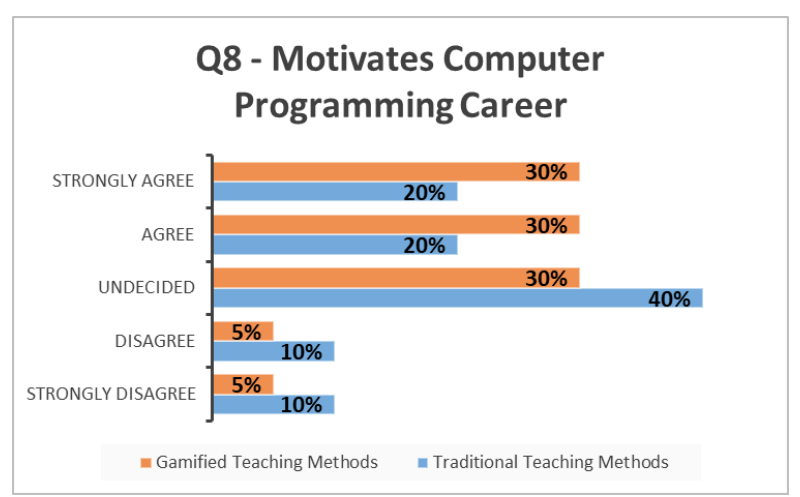

Figure 8. Q8 - Computer Programming Career 